\title{
Sorafenib-triggered radiation recall dermatitis with a disseminated exanthematous reaction
}

\author{
Dongryul Oh, MD', Hee Chul Park, MD, PhD', Ho Yeong Lim, MD, PhD², Byung Chul Yoo, MD, PhD \\ ${ }^{1}$ Department of Radiation Oncology, ${ }^{2}$ Division of Hematology and Oncology, ${ }^{3}$ Department of Internal Medicine, \\ Samsung Medical Center, Sungkyunkwan University School of Medicine, Seoul, Korea
}

\begin{abstract}
Sorafenib is a multi-targeted kinase inhibitor, which is the current standard treatment for advanced hepatocellular carcinoma (HCC). Only one case of radiation recall dermatitis (RRD) associated with sorafenib has been reported so far. Our patient with recurrent HCC was treated with palliative radiotherapy (RT) for the chest wall mass. Sorafenib at $400 \mathrm{mg}$ twice daily was begun on the day following RT. On the 14th day post-RT, an erythematous patch was observed on right chest wall which matched area previously irradiated. It was consistent with RRD. Ten days later, a disseminated exanthematous rash and severe pruritus occurred. Sorafenib was stopped and an oral antihistamine was prescribed to relieve symptoms. At the 1-week follow-up after the cessation of sorafenib, all symptoms were resolved. Physicians should be alert to this recall phenomenon as it can occur both in the skin and elsewhere and the occurrence of RRD may be unpredictable.
\end{abstract}

Keywords: Sorafenib, Radiotherapy, Radiation recall dermatitis

\section{Introduction}

Sorafenib is a multi-targeted kinase inhibitor which interferes the serine/threonine kinases Raf-1 and B-Raf, the receptor tyrosine kinase activity of vascular endothelial growth factor receptors 1, 2, and 3 and platelet-derived growth factor receptor $\beta$. Sorafenib is approved by the United States Food and Drug Administration for hepatocellular carcinoma and renal cell carcinoma and it is the current standard treatment for advanced hepatocellular carcinoma (HCC) based on two large-scale randomized clinical trials $[1,2]$. However, sorafenib is associated with many adverse effects including diarrhea, weight loss, fatigue, and hypertension. Most notably, cutaneous effects, such as rash, dryness, and hand-foot skin reaction, have been frequently reported [3]. Radiation recall dermatitis
(RRD) is defined as the 'recalling' by skin of previous radiation exposure, and is induced by the administration of certain drugs [4]. Chemotherapeutic agents, such as docetaxel, doxorubicin, gemcitabine, and paclitaxel, are commonly associated with RRD. However, only one case of RRD associated with sorafenib has been reported [5]. We report a case of RRD followed by disseminated exanthematous rash triggered by sorafenib.

\section{Case Report}

A 50-year-old man with chronic hepatitis B presented with an incidental computed tomography (CT) finding of a liver mass. He had been treated with lamivudine for chronic hepatitis B for five years. Dynamic liver magnetic resonance imaging showed an approximately $3.8-\mathrm{cm}$-sized single nodular mass with

Received 13 August 2013, Revised 6 Setember 2013, Accepted 11 Setember 2013.

Correspondence: Hee Chul Park, MD, PhD, Department of Radiation Oncology, Samsung Medical Center, Sungkyunkwan University School of Medicine, 81 Irwon-ro, Gangnam-gu, Seoul 135-710, Korea. Tel: +82-2-3410-2605, Fax: +82-2-3410-2619, E-mail: rophc@skku.edu

(c) This is an Open Access article distributed under the terms of the Creative Commons Attribution Non-Commercial License (http://creativecommons.org/ licenses/by-nc/3.0/) which permits unrestricted non-commercial use, distribution, and reproduction in any medium, provided the original work is properly cited.

www.e-roj.org 
arterial enhancement and washout on segment 5 . His serum alpha-fetoprotein level was $3.1 \mathrm{ng} / \mathrm{mL}$. Liver biopsy revealed HCC with a background of chronic active hepatitis. A chest CT showed a dense, mass-like consolidation in right upper lobe. Bronchoscopic biopsy demonstrated extranodal marginal zone B-cell lymphoma of bronchus-associated lymphoid tissue. He was treated with hepatic bisegmentectomy (segments 5-6) for HCC.

At the one-year follow-up after surgery, multiple intrahepatic recurrent masses, peritoneal seeding masses, and a right chest wall mass were detected by dynamic liver MRI. Trans-catheter arterial chemoembolization was performed for the multiple intrahepatic metastases. For the chest wall mass, palliative radiotherapy (RT) was delivered with a total dose of 39 Gy in 13 fractions. Parallel opposing fields with wedge filters were used (Fig. 1). Sorafenib at $400 \mathrm{mg}$ twice daily was begun on the day following completion of RT.

At a follow-up visit on the 14th day post-RT, the patient complained of mild pruritus of the right chest wall, and an erythematous patch with dry desquamation was observed on right chest wall which matched area previously irradiated and was consistent with RRD (Fig. 2). Because the pruritus was tolerable, sorafenib was continued at $400 \mathrm{mg}$ twice daily. He visited the hospital again 10 days later presenting with a disseminated exanthematous rash and severe pruritus (Fig. 2). Sorafenib was stopped and an oral antihistamine was prescribed to relieve symptoms. At the 1-week follow-up after the cessation of sorafenib, the pruritus and skin rash were resolved (Fig. 2). Sorafenib was started again concurrently with an oral antihistamine drug and the skin rash did not recur.

\section{Discussion and Conclusion}

There has been only one previous case report of RRD triggered by sorafenib [5] in which sorafenib was begun three weeks after RT. A hyperpigmented patch with dry desquamation matched to the irradiated field was observed 12 days after starting sorafenib. In our case, sorafenib was started on the day following completion of RT and a skin rash matched to the irradiated field developed 14 days after starting sorafenib. Actually, this skin reaction may be associated with RRD as well as acute radiation dermatitis enhanced by sorafenib (radiosensitization) because of the short time interval between $\mathrm{RT}$ and exposure to sorafenib in our case. In addition, adverse skin reaction of sorafenib alone may be related, because a disseminated exanthematous rash developed later. These three
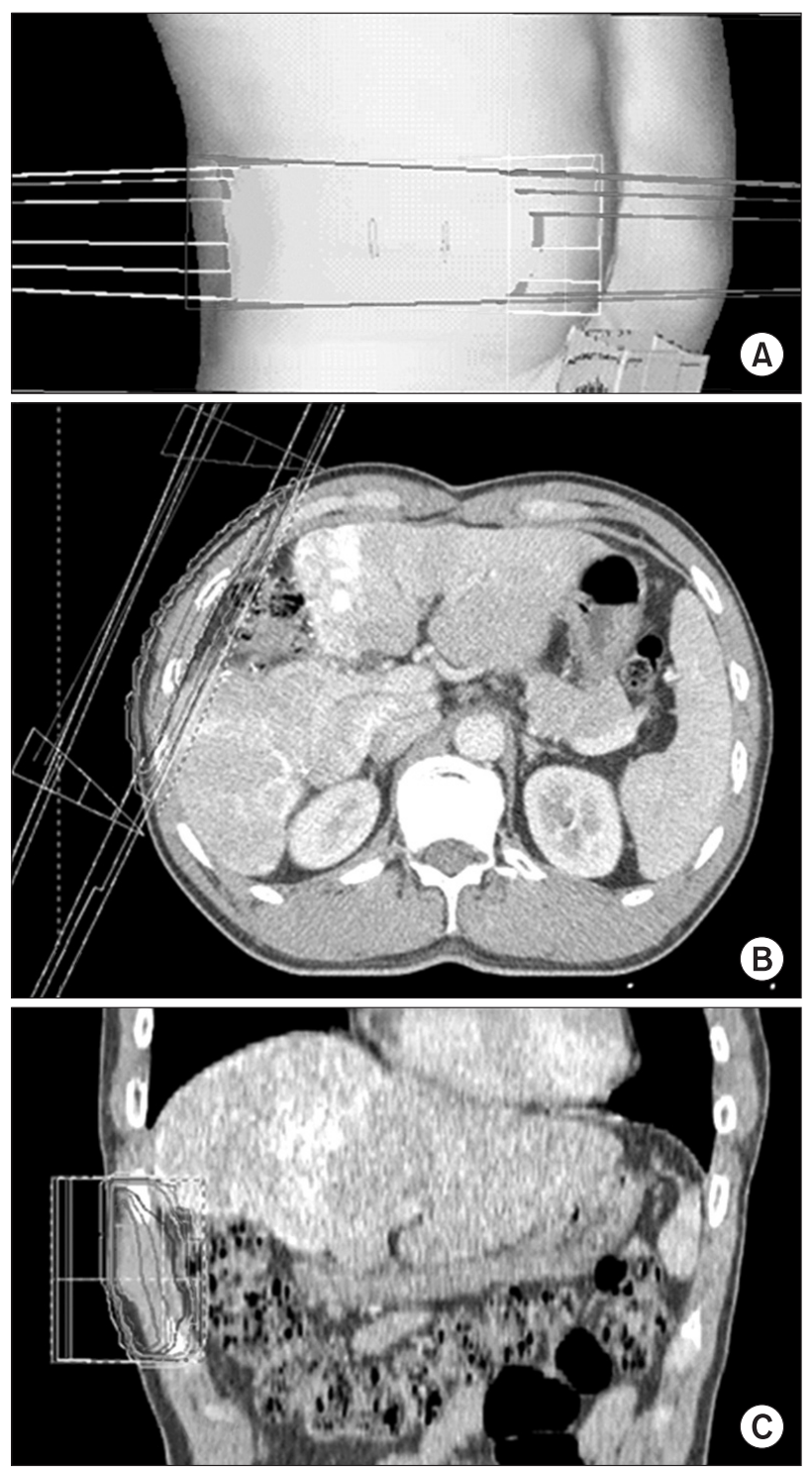

Fig. 1. Radiotherapy planning with a parallel opposing field. (A) Three-dimensional model view, (B) axial view, and (C) coronal view.

phenomena cannot be distinguished practically. We did not perform the skin biopsy, but it may be helpful. The pathologic finding of sorafenib-induced skin reaction shows varying degree of keratinocyte damage, while that of acute radiation dermatitis shows an inflammatory reaction with wound healing process [6,7]. RRD can have both features [4].

RRD was first described by D'Angio et al. [8] in 1959, and was found to be triggered by D-actinomycin. The mechanism of RRD remains unclear, although proposed explanations include a variant of a drug hypersensitivity reaction, vascular damage, or the depletion of epithelial stem cells within the irradiated field. Docetaxel, doxorubicin, gemcitabine, and paclitaxel 

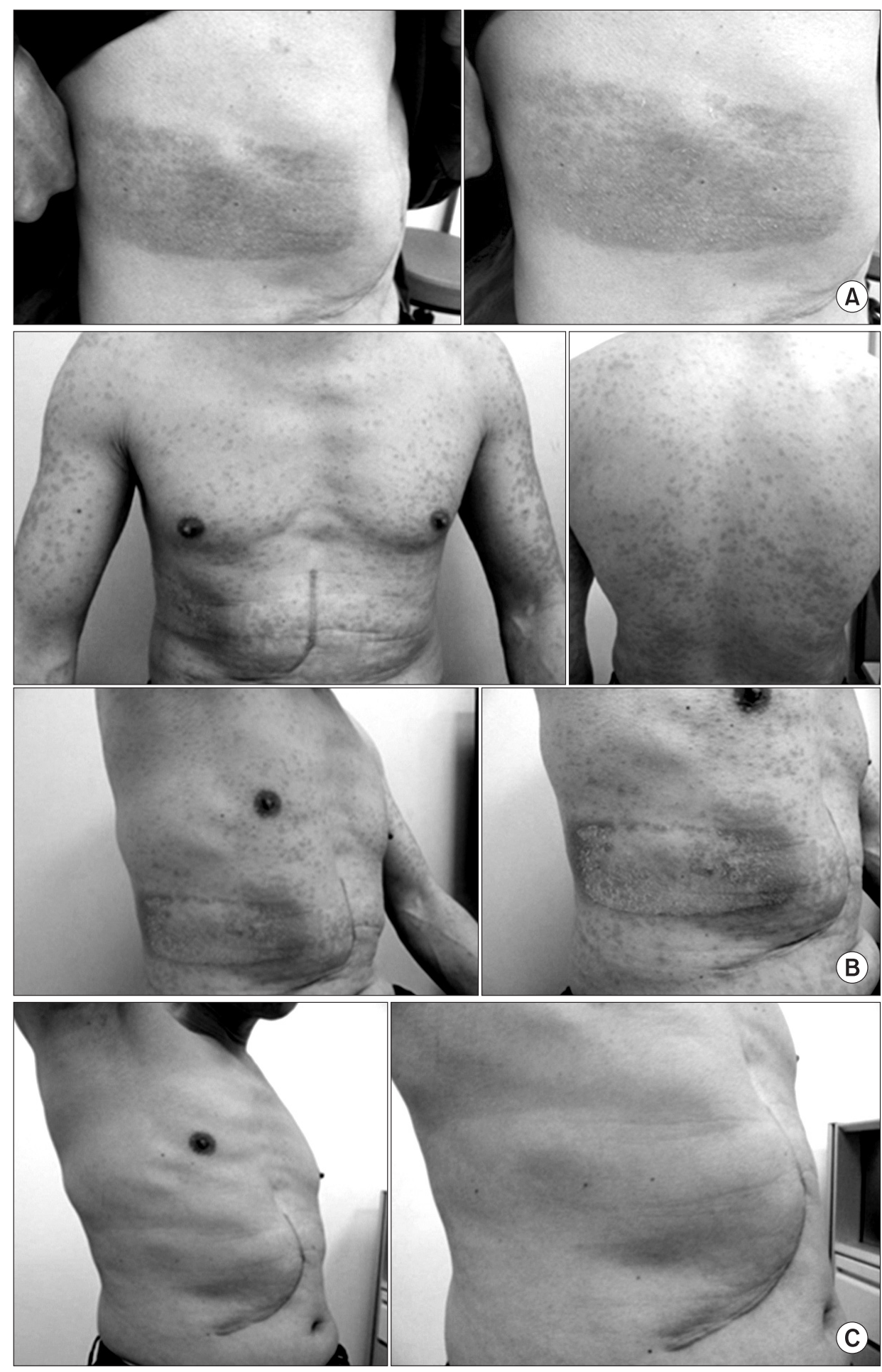

Fig. 2. Radiation recall dermatitis followed by a disseminated exanthematous rash. (A) 14 days after radiotherapy (RT), (B) 24 days after RT, and (C) 31 days after RT.

are frequently associated with RRD [9]. RRD triggered by monoclonal antibodies, such as cetuximab [10], trastuzumab $[11,12]$ has also recently been reported. The reported time intervals between RT and subsequent exposure to the RRDtriggering drug have ranged from days to years. The likelihood of developing RRD seems to be decreased as the time interval increases [13]. Camidge and Price [4] suggested that time interval between RT and subsequent chemotherapy should be longer than 7 days in RRD to exclude radiosensitization effect by chemotherapy. They found that the median time interval 


\section{Dongryul Oh, et al}

between RT and subsequent exposure to the triggering drug was 39.5 days, and time of onset of the 'recalling' skin reaction ranged from a few minutes to 14 days (median, 3 days) with intravenous drugs and from 3 days to 2 months (median, 8 days) with oral drugs. An acute skin reaction during RT is not a prerequisite for RRD. Many patients who developed RRD did not experience any acute skin reaction during radiation therapy [14]. RRD usually resolves after discontinuation of the triggering drug. The resolution time varies from a few hours to a few months [4]. Although the role of steroids in the management of RRD is unclear, they are used commonly for symptom control and for preventing recurrent "recall phenomenon" if chemotherapy is subsequently preformed [9]. The effect of dose reduction on reducing the chance of RRD recurrence also remains unknown [4]

The multi-targeted kinase inhibitor sorafenib is used for the treatment of HCC and renal cell carcinoma and is associated with a variety of cutaneous adverse effects. Hand-foot syndrome (HFS) is the typical skin reaction and is characterized by localized painful lesions on the palms and soles accompanied by hyperkeratosis or blistering [3]. Although the precise mechanism remains unknown, the proposed etiology of HFS is an increase in drug concentration in the rich capillary network and an increase in blood flow to the palms and soles, which then interferes with an unknown molecular pathway in keratinocytes and results in keratinocyte damage. The pathologic findings of HFS revealed keratinocyte vacuolar degeneration, the presence of intracytoplasmic eosinophilic bodies, and intraepidermal blisters in the stratum malpighii [7]. Facial erythema, subungual splinter hemorrhage, dry skin, and alopecia are also frequently reported [3]. Interestingly, our case presented with RRD on the chest wall first and later a disseminated exanthematous rash on the whole body. This more closely resembles a classic skin hypersensitivity reaction to drugs and the mechanism of this disseminated exanthematous rash may be different from that of HFS.

Multi-targeted kinase inhibitors, including sorafenib, have been increasingly used to treat cancer patients, and the use of these drugs in patients who have been previously irradiated will likely increase. Although few cases of RRD have been reported so far, physicians should be alert to this recall phenomenon as it can occur both in the skin and elsewhere and the occurrence of RRD may be unpredictable.
Radiation Oncology Journal ROJ

\section{Conflict of Interest}

No potential conflict of interest relevant to this article was reported.

\section{References}

1. Llovet JM, Ricci S, Mazzaferro V, et al. Sorafenib in advanced hepatocellular carcinoma. N Engl J Med 2008;359:378-90.

2. Cheng AL, Kang YK, Chen Z, et al. Efficacy and safety of sorafenib in patients in the Asia-Pacific region with advanced hepatocellular carcinoma: a phase III randomised, doubleblind, placebo-controlled trial. Lancet Oncol 2009;10:25-34.

3. Robert C, Mateus C, Spatz A, Wechsler J, Escudier B. Dermatologic symptoms associated with the multikinase inhibitor sorafenib. J Am Acad Dermatol 2009;60:299-305.

4. Camidge R, Price A. Characterizing the phenomenon of radiation recall dermatitis. Radiother Oncol 2001;59:237-45.

5. Chung C, Dawson LA, Joshua AM, Brade AM. Radiation recall dermatitis triggered by multi-targeted tyrosine kinase inhibitors: sunitinib and sorafenib. Anticancer Drugs 2010;21:206-9.

6. Hymes SR, Strom EA, Fife C. Radiation dermatitis: clinical presentation, pathophysiology, and treatment 2006. J Am Acad Dermatol 2006;54:28-46.

7. Yang $\mathrm{CH}$, Lin WC, Chuang $\mathrm{CK}$, et al. Hand-foot skin reaction in patients treated with sorafenib: a clinicopathological study of cutaneous manifestations due to multitargeted kinase inhibitor therapy. Br J Dermatol 2008;158:592-6.

8. D'Angio GJ, Farber S, Maddock CL. Potentiation of X-ray effects by actinomycin D. Radiology 1959;73:175-7.

9. Hird AE, Wilson J, Symons S, Sinclair E, Davis M, Chow E. Radiation recall dermatitis: case report and review of the literature. Curr Oncol 2008;15:53-62.

10. Law $A B$, Junor EJ. Chemotherapy-induced recall of cetuximab and radiation skin reaction. Clin Oncol (R Coll Radiol) 2009:21:77-8.

11. Chung C, Stuart D, Keyes M. Radiation recall reaction induced by adjuvant trastuzumab (herceptin). Case Report Med 2009:2009:307894.

12. Shrimali RK, McPhail NJ, Correa PD, Fraser J, Rizwanullah M. Trastuzumab-induced radiation recall dermatitis: first reported case. Clin Oncol (R Coll Radiol) 2009;21:634-5.

13. Bostrom A, Sjolin-Forsberg G, Wilking N, Bergh J. Radiation recall: another call with tamoxifen. Acta Oncol 1999;38:955-9.

14. Azria D, Magne N, Zouhair A, et al. Radiation recall: a well recognized but neglected phenomenon. Cancer Treat Rev 2005;31:555-70. 